\title{
Not Lost in Translation: Protocols for Interpreting Trauma-Focused CBT
}

\author{
Patricia d'Ardenne, Elly Farmer, Laura Ruaro and Stefan Priebe \\ Institute of Psychotrauma, East London and the City Mental Health NHS Trust, UK
}

\begin{abstract}
This paper describes the development of protocols with interpreters employed in an East London psychological trauma clinic. The service treats traumatized refugee people with cognitive methods, according to the National Institute for Clinical Excellence (NICE) Guidelines for PTSD. NICE states that language should not be a barrier to treatment, but provides no further guidance to achieve this. These protocols for interpreting CBT are designed to be explicit, consistent and ethical. A telephone poll from a recent UK-wide trauma survey, a focus group with local interpreters, and practitioner reflection were all used to develop and refine the protocols and their rationale, which are described, together with implications for future culturally sensitive practice and research.
\end{abstract}

Keywords: Interpret, cognitive-behavioural therapy, post-traumatic stress disorder, refugee people, protocols, translate.

\section{Introduction}

There is a large body of literature attesting to the high prevalence of mental health problems in refugee people ${ }^{1}$ who have suffered psychological trauma (Van de Veer 1998; Silove, Steel, McGorry and Mohan, 1998), most notably post-traumatic stress disorder (PTSD), anxiety and depression, related to the violence they have experienced in their home and adopted countries (Arthur and Ramaliu, 2000). In the UK, the National Health Service (NHS) is legally and morally required to provide a competent and culturally appropriate response to the health needs of all, with equal opportunities in receiving psychotherapy and accessing language support. The National Institute of Clinical Excellence (NICE, 2005) recommends traumafocused CBT for PTSD. It also states, differences of culture or language should not be an obstacle to the provision of effective trauma-focused psychological interventions (our italics), (Paragraph 2.3.7.3.4), but has not detailed how this should be addressed.

\footnotetext{
Reprint requests to Patricia d'Ardenne, Institute of Psychotrauma, 61 Bartholomew Close, London EC1A 7BE, UK. E-mail: patricia.dardenne@elcmht.nhs.uk

${ }^{1}$ The United Nations (UN) defines "refugee" as a person who "owing to a well founded fear of being persecuted for reasons of race, religion, nationality, membership of a particular social group or political opinion is outside the country of his [sic] nationality and is unable, or owing to such fear, is unwilling to avail himself of the protections of that country" (UN, 1951). The UN avoids the term "Asylum Seeker"; the UK government has introduced this term for political reasons. "Refugee people" in this paper includes both groups and acknowledges the personhood of those already burdened with legal labels Patel (2003).
} 
In the British NHS, interpreting may take a number of forms: the use of a bicultural or bilingual therapist; use of the client's second language; use of an advocate; bicultural mediator or interpreter. Choice will be based on the service's view of cultural appropriateness (Bhui and Olajide, 1999), the type of therapy (Patel, 2003), as well as pragmatic limits including costs (Mailloux, 2004). In 2005 an initial survey indicated that 14 out of 17 specialist NHS psychological trauma services (including this service) saw refugee people, although it was not specified whether or not they used interpreters (Jankovic Gavrilovic, d'Ardenne, Bogic, Capuzzo and Priebe, 2005). In East London, people who are experiencing traumatic stress symptoms are routinely assessed and treated here at the Institute of Psychotrauma irrespective of language or culture. The local population is transient and diverse, with over 50 languages spoken, making the use of bilingual therapists impractical, and interpreters a necessity. A culturally sensitive rather than a culturally specific model has been adopted though not yet fully evaluated (d'Ardenne, Capuzzo, Ruaro and Priebe, 2005).

At present, $60 \%$ of referrals to this service are refugee people with PTSD, and over half of these require interpreters. Additionally, there are other service users requiring interpreting who are not refugees. As with most trauma services, this clinic uses an external agency for interpreting (London Borough of Hackney). The principal intervention is trauma focused CBT (TF-CBT), facilitating the processing of traumatic memory, the restructuring of negative appraisals of the memory, and the cognitive and behavioural management of PTSD symptoms (Ehlers and Clark, 2000; Grey, Young and Holmes, 2002; Harvey, Bryant and Tarrier, 2003). Consistency in any form of psychotherapy for traumatized individuals is fundamental to building trust, safety and containment (Basoglu and Mineka, 1992; Herman, 1992; Van der Hart, Van der Kolk and Boon, 1998; Brown, Scheflin and Hammond, 1998).

Consistency applies equally to interpreted psychotherapy (Bot and Wadensjo, 2004). However, the literature provides a number of differing guidelines and models. Westermeyer (1991) identifies three models of the therapist-interpreter-patient relationship: "triangle" (wherein therapist and interpreter play an equal role), "black-box" (where the presence of the interpreter is minimized) and "bi-lingual worker" (in which the interpreter is used as a cultural consultant). Miller, Martell, Pazdirek, Caruth and Lopez (2005), in a narrative study of interpreted psychotherapy in 14 refugee torture treatment centres in the US, found that some clinicians used the "black-box" model, others adopted a "relational" approach, similar to the "triangle" model, in which the interpreter was viewed as a significant witness to the client's story. In South Africa, Drennan and Swartz (1999) describe other models; "language specialist", "culture specialist", "institutional therapist", and "patient advocate". In the UK, interpreters have been described as cultural brokers or advocates having equality within the clinical team (Tribe and Morrissey, 2004; Patel, 2003). In the Netherlands, however, Bot and Wadensjo (2004) conclude that interpreters are translators, not co-therapists or cultural mediators. Additionally, they assert, along with others (Mailloux, 2004; Shackman, 1984), that the responsibility for good interpreting lies primarily with the clinician, who must ensure the competence of the interpreter, but they concede that interpreters transform the dialogical encounter. This is consistent with Westermeyer's (1991) distinction between translation as an exchange of words with the same meaning (denotative), and interpretation, which carries suggested meaning (connotative).

There are also inconsistencies within interpreting language. Bot (2005) identified four strategies of communication in psychotherapy sessions with traumatized refugees: direct representation; direct translation; indirect representation; and indirect translation. Interpreters 
regularly did not repeat "primary turns" in direct speech despite this being the accepted international interpreting practice. Rather, they reported what the primary speakers said in a different language, followed by a direct translation, i.e., "she says, 'I am sad"'. Here, the reporting verb serves as a "space builder" between the interpreter and the primary speaker, and clarifies the communication as well as distancing the interpreter from the other two parties. However, Bot and Wadensjo (2004) argue that this use of indirect speech "does not automatically make it easier for the parties to understand one another" (p. 360) and can confuse the other parties.

With regard to symbolic language, Tribe and Morrisey (2004) argue that proverbs and metaphors in interpreted therapy are best avoided as they are culturally specific. However, Bjorn (2005) proposes that these can enrich interpreting if selected with care. Miller et al. (2005) observe that interpreters can be used as "cultural consultants" when they are able to assist the clinician and patient in understanding figures of speech. Fox (2001) argues that short-term work should be literal, but that interpreters must eventually communicate metaphor and nuance.

One area of agreement is the ethical nature of interpreted psychotherapy. Patel (2003) highlights the power imbalance in interpreted psychotherapy and argues that traumatized refugee people must be empowered so that no further abuse occurs. To achieve this, she introduces principles of strict time-keeping, clarity of role of both practitioner and interpreter, and maximum patient choice (e.g. the qualities of the interpreter and choice of language). Many refer to the use of relatives of patients as informal interpreters as a further abuse of clinical power, and an obvious transgression of patient confidentiality (Holder, 2002; Raval, 1996; Drennan and Swartz, 1999; Mahtani, 2003). Basoglu and Mineka (1992) and Basoglu (1992) note that unpunctuality and unpredictability may increase anxiety by re-enacting the severe violation of boundaries in torture, and that any imposed therapy may confirm the helplessness and disempowerment of torture. Amodeo, Grigg-Saito and Robb (1997) emphasize the need for the patient to give informed consent to interpretation. Finally, interpreters should be empowered and supported through team participation or integration, preparation and debriefing, clinical supervision and support groups (Drennan and Swartz, 1999; Tribe, 1997, 1999; Fox, 2001; Gander and Fox, 2004; Mailloux, 2004).

Trauma-focused CBT not only aims to be consistent and ethical, but also effective. Raval and Smith (2003) hypothesized that a positive alliance between therapist and interpreter would decrease drop-out rates and increase the relevance of interpreted systemic interventions with young people and families in East London. Amodeo et al. (1997) argue that following key guidelines such as reviewing the interview structure and content with the interpreter and reiterating the importance of accurate translation will lead to better therapeutic outcome, but no evidence for this is presented. In fact, there appears to be little evaluation of interpreted psychotherapy for trauma of any kind. For example, the only recent outcome paper on CBT with refugee people (Pauvonic and Ost, 2001) specifically excluded participants requiring interpreting.

In summary, the current literature does not provide consistent guidelines for how interpreting trauma-focused psychotherapy should be done, despite providing a clear ethical framework, and there is little evaluation of effectiveness. Additionally, there may be specific aspects of TF-CBT that would favour certain methods of interpreting, which have yet to be identified. This paper aims to address these limitations by describing the development of interpreting protocols for TF-CBT that are designed to be explicit, consistent and ethical. It is hoped that this will provide the first step in ensuring effective interpreting practice for TF-CBT. 


\section{Method}

In summary, preliminary interpreting protocols based on good practice (d'Ardenne et al., 2005) were already identified as a foundation document for the study. Two of the authors of an original study of UK trauma services (Jankovic Gavrilovic et al., 2005) re-contacted those services by telephone to ascertain current interpreting practice. Next, members of the local interpreting service were invited to interview. They brought with them their existing guidelines, which were then integrated with the department's preliminary protocols. Lastly, the team formally reflected on the literature, the poll and the interpreters meeting, and developed the protocols further through a recursive process. The final version of these is given in Appendix 1.

\section{UK telephone poll}

Jankovic Gavrilovic et al. (2005) surveyed 17 UK specialist trauma services. Two of the original authors re-contacted these services again by telephone and e-mail. They enquired, first, if they provided CBT for their patients with PTSD, and whether they also offer interpreted CBT? Second, they asked what interpreting protocols, if any, they used to guide their clinical practice? These were compared with the Institute's preliminary protocols for interpreting, which had little detail.

\section{Interpreters' focus group}

The director of the interpreting service used by the clinic, and three of her most experienced interpreters were invited by the authors to the Institute. They were asked to bring any of their own professional protocols in use. The purpose of the meeting was to help identify consistent good practice in working with traumatized patients. The meeting was organized as a one and a half hour semi-structured interview. It covered the following themes: good mental health interpreting, good practice in interpreting trauma-focused CBT, and barriers to achieving this. The meeting was audio-taped with the consent of the interpreters and transcribed by the authors for accuracy. Copies of the protocols for interpreters were kept by the authors, and modified in the light of the concerns the interpreters themselves raised during the focus group. These were added and integrated with the draft protocols of the Institute.

\section{Team reflection}

The authors invited the team to allocate half a day for reflection on the telephone poll, the contributions of the interpreters, relevant literature, the developing clinical protocols, feedback from service users, and their observations of current and past clinical practice. The relevant literature was identified through a structured literature search (Figure 1), which was conducted following the recommendations of QUOROM (http://www.consortstatement.org/QUOROM.pdf). The results of the literature search (including websites such as PILOTS http://www.ncptsd.vs.gov.uk//publications/pilots/index.html) were organized into the sections as they were emerged thematically. Additionally, clinicians were asked to identify interpreted work within trauma-focused CBT judged to have been ethical and to have achieved good outcome. The team reflection was transcribed and then used to inform the development and fine tuning of the protocols. 


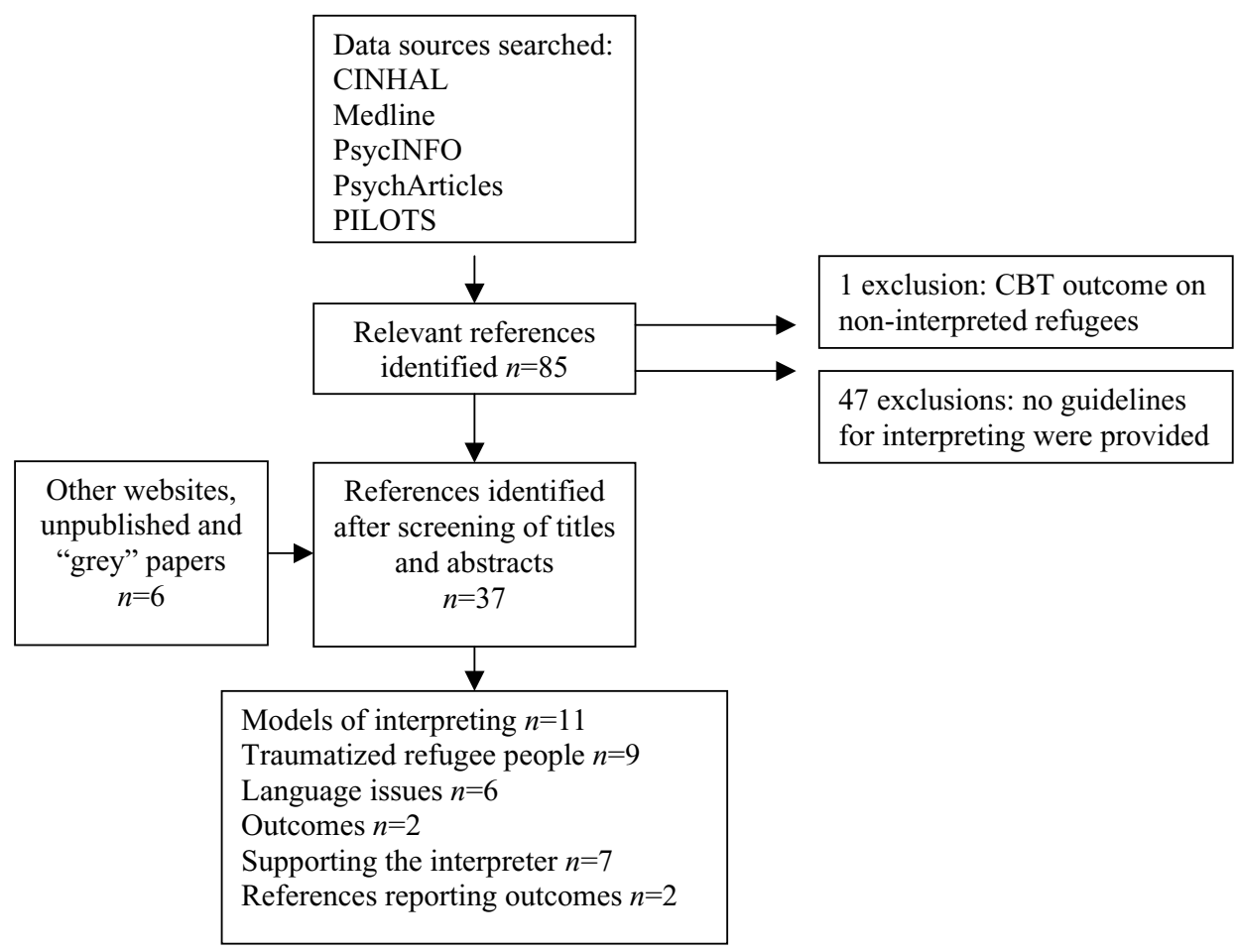

Figure 1. Results of the literature review on interpreting guidelines based on the QUOROM model (http://www.consort-statement.org/QUOROM.pdf)

\section{Results}

\section{Telephone poll}

Of the 19 NHS services contacted, 17 reported that they carry out interpreted psychotherapy; 16 of these provided CBT, and all of these used interpreters for trauma focused therapy. Fifteen employed interpreting services from their local authority; none employed interpreters directly. Four services occasionally used Language Line, an independent telephone interpreting service. All indicated that, to the best of their knowledge, local interpreters worked to professional interpreting guidelines, but that the NHS mental health services themselves did not provide these. One service provided generic mental health training for its interpreters. No service had specific interpreting protocols for mental health or for CBT. Eight services commented that additional time was allocated to interpreters at the end of treatment sessions for comments and feedback, and that this was deemed helpful.

\section{Interpreters' focus group}

The interpreters brought their own published general guidelines, which were incorporated into the discussion and overall conclusions and which contributed to the developing CBT protocols. The service uses a "linguistic" model of consistency and impartiality, requiring as 
literal an interpretation as possible, without discussion, opinion sharing, or advocacy. Pertinent guidelines are summarized below:

- Being a good listener and clear communicator

- Being inclusive and interpreting everything including material that makes no sense or has only partial meaning

- Demonstrating tolerance and understanding of the stress of mental disorder

- Understanding the meaning of confidentiality in mental health

- Advising the clinician to simplify or re-present questions for the client

- Not adding or omitting any material

In addition, the interpreters generated requirements for interpreters working with therapists practising trauma-focused CBT as summarized below:

- Knowledge of post traumatic stress disorder, and its main groups of symptoms

- Knowledge of trauma and CBT terminology and vocabulary

- A working knowledge of the current treatments for post traumatic stress disorder

- Specific rationale for re-visiting trauma history in assessment and CBT treatment

- An extensive emotional vocabulary

The interpreters described a range of personal issues and challenges in interpreting trauma focused CBT, listed below.

- "Emotional baggage", by which they meant that interpreters' own emotional distress and what they brought into the consultation room. One cited the specific example of hearing about the killing of a young girl who was the same age as that of the interpreter's daughter. The interpreter identified with the victim and became tearful when she went home that night.

- "Emotional unpreparedness". Interpreters felt overwhelmed with the intensity of traumatic material being discussed, and were fearful of the terror and horror of patients' experiences revealed during CBT assessment and reliving sessions.

- Lack of emotional detachment. Some interpreters had opted out of mental health work because they had found this difficult to achieve. Interpreters described the importance of seeing their work as "just a job" and not becoming too involved with what was happening in the treatment room.

- Fear of re-traumatization. Some interpreters had also experienced the traumatic events of crime, imprisonment, war and torture. Listening to the patients' stories repetitively and in detail might within CBT place them at risk of developing mental health problems. They also believed that the patient would be re-traumatized by this process.

- Occupational "burn-out". When the clinic booked interpreters of one language with a number of patients back to back (for reasons of economy), the interpreters felt overwhelmed and stressed.

Solutions suggested by the interpreters are listed below:

- A briefing on traumatic memory, and the development of post traumatic disorders

- Information about CBT and how it could be applied to PTSD would help professional interpreting prior to seeing any single patient. Few of the interpreters were aware of the models being used by clinicians 
- More information about culture of the patient and language support needs prior to accepting a booking for the service

- A briefing session just before the first appointment on client's background was requested. A brief trauma history would prepare interpreters emotionally for the session, and permit them to check any issues for mental preparation - traumatic, cultural or linguistic. They did not specify the length of this briefing session

- De-briefing at end of all sessions - duration unspecified

- Allocation of time between patients within one clinic

- A separate waiting area for confidentiality and to prevent further disclosures from patient to interpreter outside the session

\section{Team reflection}

Contributions from the clinical team were based on the results of the telephone poll, the meeting with the interpreting service, copies of the literature search, the draft protocols, and current clinical practice and observations. The contradictions in the literature were discussed and resolved. Criteria for the protocols included how each would "fit" with CBT, pragmatic constraints of a small local service, NHS clinical governance and ethical practice (BPS Ethical Guidelines, 2006). Clarity, simplicity and practicality were important criteria. Pertinent themes and conclusions are outlined below.

General interpreting model. The team opted for a literal, sequential interpreting model, using direct speech in the first and second person with a specific seating plan for this purpose. This model was chosen to maximize communication in an exchange where meaning is always transformed and relative (Bot and Wadensjo, 2004). This was a more realistic model for interpreters from an outside agency untrained in CBT. The team concluded that the clinician would always have responsibility for higher levels of communication of meaning (Mailloux, 2004). The team also reflected on how concerns about refugees' experience of interrogation (Patel, 2003; Burnett and Peel, 2001) might be addressed within the above model. It was agreed that some flexibility would need to be shown with seating arrangements, eye contact and confidentiality.

Interpreting demands specific to trauma-focused CBT. A significant number of interpreters working in East London themselves come from the refugee communities seen in this clinic. Some have witnessed the atrocities of war, imprisonment and even torture. CBT work with traumatic memory requires a detailed revisiting of life-threatening violence, which may be a particularly demanding task for an interpreter historically involved in such events. In addition, the paradoxical nature of trauma-focused work (i.e. remembering, not forgetting) understandably leads some interpreters to want to "protect" the patient during re-visiting. The team recommended educating interpreters about the nature and rationale of this work, and selected a booklet by Herbert (2002) as a clear and brief introduction to the subject. The team reflected on how metaphors communicate the rationale of trauma focused work and CBT. It was agreed that clinician and interpreter needed to choose those that are culturally appropriate as well as capable of interpretation, prior to or subsequent to the clinical session.

The team judged that TF-CBT requires the accurate identification and representation of traumatic memory, including all the nuanced meaning of emotional "hotspots". Therefore interpreters would have to provide more than a literal word-for-word account for traumatic 
memory. Temporal proximity of phrases between clinician and client during reliving was deemed to be critical in ensuring imaginal exposure to the past and, because of this, shorter "chunks" were recommended. The team recognized the value of consistency and clarity in all its interpreted work with PTSD, as recommended by the NICE guidelines for CBT, and reflected on how protocols could be incorporated into a contract between clinical and interpreting services (Amodeo et al., 1997).

Ethics. The team agreed that consistency is integral to trust and safety with all trauma survivors, and that the development of protocols would lead to greater reliability, transparency and accountability in its clinical practice. Consistency contrasts with the unpredictable and uncontrollable life events patients have survived, (Basoglu, 2004). East London is a microcosm of the world's communities (d'Ardenne et al., 2005) and interpreters come from these. Some ethnic groups are small, and interpreters from these communities inevitably meet patients in both healthcare and social settings, which brings particular challenges. The team decided on a general model that would protect the boundaries of its interpreters and the confidentiality of clients.

The literature (Tribe, 1997, 1999; Drennan and Schwartz, 1999; Patel, 2003) refers to the empowerment of interpreters and the protection of their well-being. The team generated means of achieving both these objectives within the clinical setting and with the interpreter service through a contract, supported by regular peer supervision group. Informed consent to interpreting is a means of empowering patients, and the team agreed to include consent to interpreting within its opt-in letter to the trauma service; this had previously not been explicit.

The authors wrote a final version of the protocols based on all the results, which are listed in Appendix I. They are brief, simple and practical and are divided into three sections: the service, the clinician and the interpreter. The protocols reflect the following principles, which comprise an interpreting model for TF-CBT.

1. The overall responsibility of the clinician for good interpreting

2. The deployment of interpreters within a practitioner-led therapy

3. The education of interpreters about the TF-CBT model

4. To brief interpreters on the paradoxical nature of trauma-focused work

5. To ensure the interpreters' well-being within TF-CBT

6. To use interpreters as cultural consultants

7. To maximize cognitive and emotional meaning across languages

\section{Discussion}

This paper has described the development of protocols for interpreting CBT with traumatized people through a number of stages. The rationale for the study was that despite the recommendations of NICE to provide interpreted CBT, there appear to be no guidelines to support current practice. Across the UK, specialist trauma centres appear to be seeing refugees without any shared or explicit understanding of how interpreting is provided. A literature search provided recommendations about ethics and practice of interpreting in mental health, but these were neither specific nor consistent, and there was nothing located on interpreting trauma-focused CBT. The authors listened to the concerns of their own interpreting service through a semi-structured interview and through team reflection as a process for preparing protocols for trauma focused interpreted CBT. 
The literature on interpreting psychotherapy provided little evidence on interpreted therapy outcomes, nor on the perceptions of clients receiving it. The quality of interpreting may depend on the presenting problems in therapy, the model of therapy, the model of interpreting, and the philosophy of the service regarding the nature of verbal communication. The literature on refugee people varies in its philosophy and analysis, and some have argued against the application of Western models to any refugee distress (Summerfield, 2002). Nobody would dispute that people whose rights have been extremely violated have complex needs, and are entitled to human rights support. But it remains the case that effectively interpreted health care for those who may have suffered the trauma of extreme physical and mental violence is an integral part of their human rights, not an alternative to it.

The interpreters' focus group provided the first opportunity to address specific issues in working with trauma focused CBT, and for understanding and resolving professional and pragmatic requirements within this service from different perspectives. Interpreters in the service were experienced but had little mental health training. The interpreters were consistent in their requests for more information about CBT and the rationale behind re-visiting traumatic memory, echoing the concerns of patients. A self-help booklet used for patients (Herbert, 2002) was selected as appropriate for them, because it provides a brief and clear account of PTSD and its effects on the individual, and the rational and application of cognitive therapy.

Team reflection provided a psychological space for the clinicians who see traumatized people requiring language support for assessment and therapy. They receive training and support in cultural competency in their clinical practice, as well as weekly supervision and on the job learning for their work with interpreted CBT. Two of the team have worked with overseas non-governmental agencies (NGOs) serving refugee populations; one is a qualified interpreter; the team also collects routine outcome data for interpreted and non-interpreted CBT with PTSD patients.

A model of interpreting has been developed that is consistent with the principles and methods of CBT. Such a model is not necessarily based on common sense or even current practice; rather it is the integration of a wide range of ideas and ideals, which have yet to be evaluated. The ethical literature consistently upholds the empowerment of the client. However, the debate exists between those who seek empowerment of the interpreter as a team player, or as being accountable to the clinician. The answer may be determined by whether or not interpreters are employees of the clinical service and are subject in the same way as practitioners to clinical governance within the NHS. These guidelines cover the pragmatic realities of interpreters with minimal mental health training who are employed from external agencies. They clearly place responsibility for interpreted CBT with the clinician and yet give interpreters enough guidance to work confidently and consistently. They can therefore be adapted to other psychotherapy services employing external interpreters with a wide range of experience.

The limitations of this study include the small numbers of services surveyed, the limited literature in the field, and the small numbers of interpreters and clinicians involved. A particular omission was the perspectives of service users, their families and communities (Burnett and Peel, 2001). Unfortunately, there is also little in the literature that informs us about users' perceptions and wishes in interpreting. Similarly, referrers to this specialist service were not consulted about interpreted CBT. Lastly, the authors have yet to explore the established practice of interpreting in NGO's working with traumatized communities.

Williams, Bradshaw, Fournier, Bray and Hodson (2005) point out the need for protocols and for systems that are capable of being evaluated and these brief protocols represent that first 
step. This was a modest study that has developed protocols whose contribution to improving CBT outcome has yet to be demonstrated. The next stage entails applying them to service users, interpreters, other clinicians, and other services facing similar clinical issues. There will be a period of evaluation, adjustment and new application. Future research will need to identify further how TF-CBT has to be modified when an interpreter is present. We hope that these protocols help to overcome cultural and language barriers for traumatized patients in need of effective psychological care.

\section{Acknowledgements}

The authors would like to thank the following: Rachel Holder whose dissertation and generosity was an inspiration for this paper; the interpreters of the London Borough of Hackney Interpreting service, and in particular its Director, Dina Cain, for her valuable input and comments; to Alison Hauenstein Swan and Graham Fawcett for the team feedback and, lastly, Leticia Cestari and Carleen Scott for their assistance with the research.

\section{References}

Amodeo, M., Grigg-Saito, D. and Robb, N. (1997). Working with foreign language interpreters: guidelines for substance abuse clinicians and human service practitioners. Alcoholism Treatment Quarterly, 15, 75-87.

Arthur, N. and Ramaliu, A. (2000). Crisis intervention with survivors of torture. Crisis Intervention, $6,51-63$.

Basoglu, M. (Ed.) (1992). Torture and its Consequences, Current Treatment Approaches. Cambridge: Cambridge University Press.

Basoglu, M. (2004). Cognitive Behavioural Therapy with Refugees. Proceedings of the XXIV Annual Congress of the European Association for Behavioural and Cognitive Therapies, September. Manchester: University of Manchester.

Basoglu, M. and Mineka, S. (1992). The role of uncontrollable and unpredictable stress in post-traumatic stress responses in torture survivors. In M. Basoglu (Ed.), Torture and its Consequences, Current Treatment Approaches. Cambridge: Cambridge University Press.

Bhui, K. and Olajide, D. (Eds.) (1999). Mental Health Service Provision for a Multicultural Society. London: WB Saunders.

Bjorn, G. J. (2005). Ethics and interpreting in psychotherapy with refugee children and families. Nord Journal of Psychiatry, 59, 516-521.

Bot, H. (2005). Dialogue interpreting as a specific case of reported speech. Interpreting, 7, 237-261.

Bot, H. and Wadensjo, C. (2004). The presence of a third party: a dialogical view on interpreter-assisted treatment. In J. P. Wilson and B. Drozdec. (Eds.), Broken Spirits: the treatment of traumatised asylum seekers, refugees, war and torture victims. East Sussex: Brunner Routledge.

Brown, D., Scheflin, A. W. and Hammond, D. C. (1998). Memory, Trauma Treatment and the Law. New York: Norton.

Burnett, A. and Peel, M. (2001). Health needs of asylum seekers and refugees. British Medical Journal, 322, 544-547.

D'Ardenne, P., Capuzzo, N., Ruaro, L. and Priebe, S. (2005). One size fits all? Cultural sensitivity in a psychological service for traumatised refugees. Diversity in Health and Social Care, 2, 29-36.

Drennan, G. and Swartz, L. (1999). A concept overburdened: institutional roles for psychiatric interpreters in post-apartheid South Africa. Interpreting, 4, 169-198.

Ehlers, A. and Clark, D. (2000). A cognitive model of posttraumatic stress disorder. Behaviour Research and Therapy, 38, 319-345. 
Fox, A. (2001). An interpreter's perspective. Medical Foundation Series. Medical Foundation website downloaded April 2006. This article first appeared in Context, 54, 19-20 (the magazine for family therapy and systemic practice).

Gander, J. and Fox, A. (2004). Supporting victims of torture. Medical Foundation Series, Medical Foundation website downloaded April 2006. This article first appeared in The Linguist, 43.

Grey, N., Young, K. and Holmes, E. (2002). Cognitive restructuring within reliving: a treatment for peri-traumatic emotional "hotspots" in posttraumatic stress disorder. Behavioural and Cognitive Psychotherapy, 30, 37-56.

Harvey, A. G., Bryant, R. A. and Tarrier, N. (2003). Cognitive behaviour therapy for posttraumatic stress disorder. Clinical Psychology Review, 23, 501-522.

Herbert, C. (2002). Understanding Your Reactions to Trauma: a guide for survivors of trauma and their families (rev. ed.). Oxford: Blue Stallion Publications.

Herman, J. L. (1992). Trauma and Recovery. New York: Basic Books.

Holder, R. (2002). The Impact of Mediated Communication on Psychological Therapy with Refugees and Asylum Seekers: practitioners' experiences. MSc Dissertation, City University, London.

Jankovic Gavrilovic, J., d'Ardenne, P., Bogic, M., Capuzzo, N. and Priebe, S. (2005). Survey of specialised UK traumatic stress services. Psychiatric Bulletin, 29, 416-418.

Mahtani, A. (2003). The right of refugee clients to an appropriate and ethical psychological service. International Journal of Human Rights, 7, 40-57.

Mailloux, S. (2004). Ethics and interpreters: are you practising ethically? Journal of Psychological Practice, 10, 37-44.

Miller, K. E., Martell, Z. L., Pazdirek, L., Caruth, M. and Lopez, D. (2005). The role of interpreters in psychotherapy with refugees: an exploratory study. American Journal of Orthopsychiatry, 75, 2739.

National Institute for Clinical Excellence (NICE) (2005). Post-Traumatic Stress Disorder (PTSD): the management of PTSD in adults and children in primary and secondary care. London: NICE. www.nice.org.uk (accessed 6 February 2006).

Patel, N. (2003). Speaking with the silent: addressing issues of disempowerment when working with refugee people. In R. Tribe and H. Raval. (Eds.), Working with Interpreters in Mental Health. East Sussex: Brunner Routledge.

Paunovic, N. and Ost, L. (2001). Cognitive-behaviour therapy vs exposure therapy in the treatment of PTSD in refugees. Behaviour Research and Therapy, 39, 1183-1197.

PILOTS (2006). http://www.ncptsd.va.gov//publications/pilots/index.html (Accessed 12 September).

QUOROM (2006). http://www.consort-statement.org/QUOROM.pdf. (Accessed 12 September).

Raval, H. (1996). A systemic perspective on working with interpreters. Clinical Child Psychology and Psychiatry, 1, 29-43.

Raval, H. and Smith, J. A. (2003). Therapists' experiences of working with language interpreters. International Journal of Mental Health, 32, 6-31.

Shackman, J. (1984). The Right to Be Understood: a handbook on working with, employing and training community interpreters. Cambridge: National Extension College.

Silove, D., Steel, Z., McGorry, P. and Mohan, P. (1998). Psychiatric symptoms and living difficulties in Tamil asylum seekers: comparisons with refugees and immigrants. Acta Psychiatrica Scandinavica, 97, 175-181.

Summerfield, D. (2002). Commentary on Tribe, R., Mental health of refugees and asylum seekers. Advances in Psychiatric Treatment, 8, 247-248.

Tribe, R. (1997). A critical analysis of a support and clinical supervision group for interpreters working with refugees located in Britain. Groupwork, 10, 196-214.

Tribe, R. (1999). Bridging the gap or damming the flow? Some observations on using interpreters/ bicultural workers when working with refugee clients, many who have been tortured. British Journal of Medical Psychology, 72, 567-576. 
Tribe, R. and Morrissey, J. (2004). Good practice issues in working with interpreters in mental health. Intervention, 2, 129-142.

United Nations (1951). United Nations Convention on the Status of Refugees. Geneva: United Nations.

Van Der Hart, O., Van der Kolk, B. and Boon, S. (1998). Treatment of dissociative disorders. In J. D. Bremner and C. R. Marmar (Eds.), Trauma, Memory and Dissociation (pp. 253-283). Washington, DC: American Psychiatric Press.

Van de Veer, G. (Ed.) (1998). Counselling and Therapy with Refugees and Victims of Trauma (2nd ed.). New York: John Wiley.

Westermeyer, J. (1991). Working with an interpreter in psychiatric assessment and treatment. Journal of Nervous and Mental Disease, 178, 745-749.

Williams, M., Bradshaw, C., Fournier, Tachble, A., Bray, R. and Hodson, F. (2005). The call-centre: a child welfare liaison program with immigrant serving agencies. Child Welfare, 84, 725-746.

\section{Appendix 1. Interpreting protocols for trauma focused CBT}

\section{Introduction}

Interpreting is a valuable service for patients. Our work entails trauma focused assessment and cognitive behavioural therapy, and these guidelines aim to achieve greater consistency and effect. Thank you all for your collaboration in reading them.

\section{The service}

S.1. Ensures that all interpreters and clinicians have read these protocols, and the booklet Understanding your Reactions to Trauma (Herbert, 2002).

S.2. Obtains patients' informed consent to interpreted CBT, together with gender, linguistic and cultural preferences in initial contact letter, ideally in patients' first language.

S.3. Admits advocate or named interpreter on patient's request, but prevents family members interpreting.

S.4. Sends interpreted written materials whenever possible, including information about transport, childcare and timekeeping.

S.5. Provides a separate waiting area for interpreters wherever practical.

S.6. Schedules breaks between clients in agreement with interpreter.

S.7. Convenes regular meetings between clinicians and interpreting service about quality and review of interpreting protocols.

S.8. Undertakes regular literature updates on interpreted psychotherapy. 


\section{The clinician}

C.1. Ascertains the language support needs of the patient.

C.2. Books more time: up to twice as long as non-interpreted patients for first appointment; up to half extra time for all subsequent appointments.

C.3. Briefs the interpreter about the patient and trauma history before the first session. Checks interpreter has fluent English and adequate psychological technical vocabulary, and identifies any potential difficulty or distress for the interpreter. Warns the interpreter of the distressing and emotional nature of trauma focused work. Reschedules and books another interpreter if there are unresolved difficulties. Informs interpreting service and advises the patient immediately.

C.4. Introduces the interpreter to the patient explaining the role, and agrees titles with all parties.

C.5. Sits the patient with the interpreter just behind at an angle. Ensures eye contact with both interpreter and patient. Checks that the patient is happy with this and, if not, rearranges a conventional three way seating plan.

C.6. Uses simple, direct sentences delivered at a steady pace, addressing the patient always in the second person.

C.7. Delivers speech in brief phrases particularly during reliving, and interjects if any contribution appears too long for accurate interpreting.

C.8. Records all interpreted material, the name of the interpreter and the language spoken in the patient notes.

C.9. Selects metaphors and figures of speech for their cultural and situational relevance, and checks with the interpreter.

C.10. Observes eye contact, body language, intonation, and duration of interpreted phrases by patient and interpreter. If in doubt, asks the patient to repeat what has just been interpreted.

C.11. Pauses the session if communication breaks down or interpreting stops for any reason. Explains to the patient what is happening.

C.12. Shows written results of interpreted psychometrics to both parties and checks for accuracy.

C.13. Agrees availability of all for future appointments and, if appropriate, asks the interpreter to write dates in appointment card. Escorts the patient out of the clinic seeking, if appropriate, feedback about the interpreting.

C.14. Returns to the interpreter for debriefing, clarification and feedback on language, culture, and communication.

C.15. Books the same interpreter or changes the interpreter after consultation with the interpreting service.

\section{The interpreter}

T.1. Reads these protocols and booklet supplied before an initial booking.

T.2. Arrives punctually with paperwork for a briefing with the clinician before a first appointment. 
T.3. Abides by NHS rules of confidentiality. Declines patients known socially to self or family.

T.4. Immediately informs the clinician of any work judged to be too difficult.

T.5. Sits in therapy slightly behind the patient. Speaks to the patient's ear whilst maintaining eye contact with the therapist and encourages the patient to do the same.

T.6. Interprets directly and ideally in the first person.

T.7. Interprets small sections of speech consecutively, allowing the clinician time to process and record material. This may mean gently interrupting.

T.8. Chooses words as close to the original language as possible, with the exception of figures of speech where meaning should be conveyed.

T.9. Pauses the session if material cannot be interpreted for whatever reason, and shares and clarifies problems with everyone.

T.10. Includes all repetitions, ambiguity, omissions, and disorders of speech both in form and content.

T.11. Interprets with impartiality difficult or embarrassing material (including complaints about interpretation).

T.12. Avoids interjections, even when the client expresses high levels of distress, and maintains eye contact with the clinician.

T.13. Remains with therapeutic silences.

T.14. Shares cultural meanings or contradictions with the clinician, after the patient has left, together with any other concerns about the interpreting process. 